\title{
Cockroaches Keep Predators Guessing by Using Preferred Escape Trajectories
}

\author{
Paolo Domenici, ${ }^{1, *}$ David Booth, ${ }^{2}$ Jonathan M. Blagburn, ${ }^{3}$ \\ and Jonathan P. Bacon ${ }^{2}$ \\ ${ }^{1}$ Istituto per l'Ambiente Marino Costiero \\ Consiglio Nazionale delle Ricerche \\ Localita Sa Mardini \\ 09072 Torregrande-Oristano \\ Italy \\ ${ }^{2}$ Sussex Centre for Neuroscience and School of Life Sciences \\ University of Sussex, Falmer \\ Brighton BN1 9QG \\ UK \\ ${ }^{3}$ Institute of Neurobiology \\ University of Puerto Rico \\ San Juan, PR 00901 \\ USA
}

\section{Summary}

Antipredator behavior is vital for most animals and calls for accurate timing and swift motion. Whereas fast reaction times [1] and predictable, context-dependent escape-initiation distances [2] are common features of most escape systems, previous work has highlighted the need for unpredictability in escape directions, in order to prevent predators from learning a repeated, fixed pattern [3-5]. Ultimate unpredictability would result from random escape trajectories. Although this strategy would deny any predictive power to the predator, it would also result in some escape trajectories toward the threat. Previous work has shown that escape trajectories are in fact generally directed away from the threat, although with a high variability [5-8]. However, the rules governing this variability are largely unknown. Here, we demonstrate that individual cockroaches (Periplaneta americana, a much-studied model prey species [9-14]) keep each escape unpredictable by running along one of a set of preferred trajectories at fixed angles from the direction of the threatening stimulus. These results provide a new paradigm for understanding the behavioral strategies for escape responses, underscoring the need to revisit the neural mechanisms controlling escape directions in the cockroach and similar animal models, and the evolutionary forces driving unpredictable, or "protean" [3], antipredator behavior.

\section{Results and Discussion}

When cockroaches (Periplaneta americana) encounter a sudden air movement, such as that generated by an approaching predator, they show an escape response using a large range of turning angles from their original body orientation $[6,10]$, in line with observations on animals from other taxa [7]. Here, we examine which of the following patterns of variability is observed in the trajectories of cockroach escape responses: (1) a wide unimodal distribution, (2) a random distribution, or (3) a multipeak distribution of preferred escape trajectories.

The first series of experiments tested which of these three distribution patterns of escape trajectories occurs at the individual level. Five individual cockroaches (Periplaneta americana) were startled 75-93 times each by a wind stimulus and their escape responses were video recorded (see Supplemental Experimental Procedures available online). Wind stimuli were delivered from a variety of directions, resulting in even distributions of wind angle (defined as the angle between the direction of the wind stimulus and the orientation of the cockroach one frame before their escape response; Figure 1A) (see Experimental Procedures and Supplemental Data). Responses to left and right stimuli were pooled as if all stimuli were from the right, i.e., all wind angles were between $0^{\circ}-180^{\circ}$. Cockroaches reacted to the wind stimulus by turning, followed by forward motion. Escape trajectories (ETs) were calculated as the angle between wind direction and the direction of motion of the cockroach at the end of the turning component of the escape response (Figures $1 \mathrm{~A}$ and 1B) and were treated as a circular variable (see Experimental Procedures [15]). Although previous work (e.g., [6, 10]) was commonly analyzed with $\mathrm{x}-\mathrm{y}$ linear plots of body turn against wind angle, this representation of the data reflects the assumption that the cockroach's escape is a "hard-wired," reflexive body turn that is directly related to the wind angle it detects. However, this type of analysis does not allow a direct test of the hypothesis that cockroaches may escape at fixed directions relative to the stimulus, in contrast to a circular analysis that does.

Despite some small individual variation, the ET distributions of each animal are multimodal, with at least four peaks at approximately $90^{\circ}, 120^{\circ}, 150^{\circ}$, and $180^{\circ}$, suggesting that cockroaches do indeed have preferred ETs (Figures 1C-1G). The distributions of these ETs are not significantly different among individuals $\left(\chi^{2}=27.75 ; p>0.1\right.$; d.f. $=20$; Figure 1$)$. To determine quantitatively the angular position and the number of ET peaks, we fitted mixtures of multiple Gaussian curves to the unbinned data using a maximum likelihood method (see Supplemental Data), with the constraints of equally spaced peaks with equal widths in order to minimize the number of parameters.

In four out of five cases (Figures 1C, 1D, 1E, and 1G), four ET peaks were detected in the approximate range $90^{\circ}-180^{\circ}$ (Akaike weights $0.67-0.93, \chi^{2}$ test comparing the theoretical and the experimental distributions show no significant differences with $p$ value ranging from $0.10-0.85$ ). In a fifth case (Figure $1 \mathrm{~F}$ ), the four observable peaks for this individual appear to be similar, in terms of their angular positions, to the other individuals and the distributions are not different statistically. However, the peaks in Figure 1F are less well defined, and although a multipeak fit has a higher likelihood, it is rejected as too complex with the more parsimonious corrected Akaike information criterion (AIC). Overall, the fact that almost identical peaks can be detected independently in four out of five separate animals provides very convincing evidence for the existence of a multiple-ET escape strategy. 
A

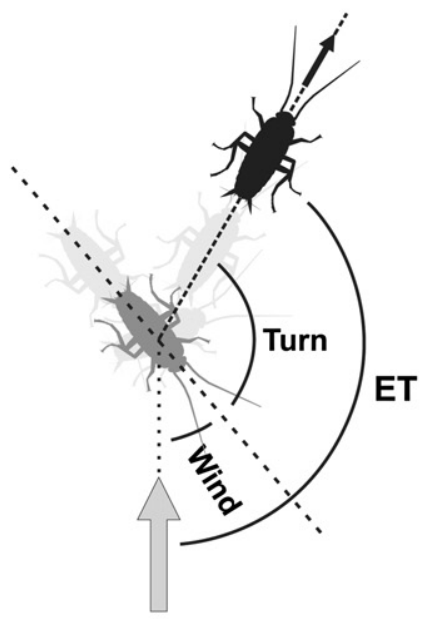

B

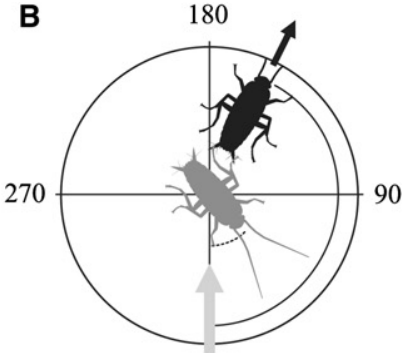

0,360

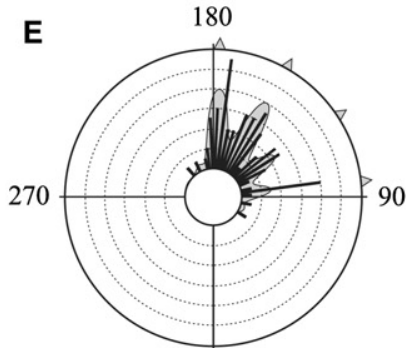

0,360
C

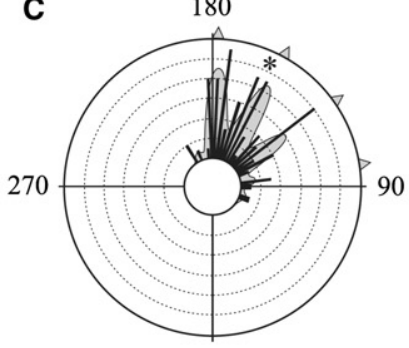

0,360

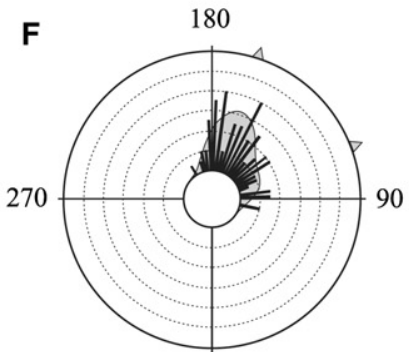

0,360

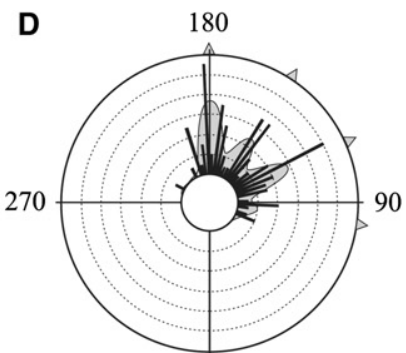

0,360

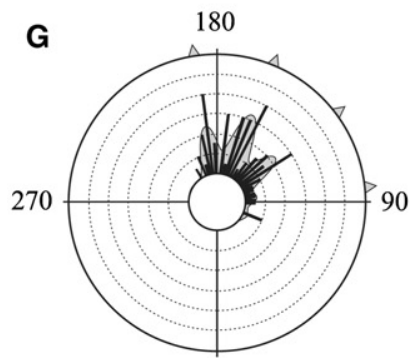

0,360

Figure 1. Individual Cockroaches Tested in Repeated Trials Show Similar, Striking Multimodal Distributions of ETs

(A) Diagram of the sequence of movements in a typical cockroach escape response. The cockroach is walking from the left to the right of the picture. The gray arrow indicates stimulus direction. Wind angle (wind) at the time of stimulation, body turn (turn), and escape trajectory (ET) are shown.

(B) Diagram illustrating the definition of escape trajectory (ET) and the way it is plotted in all subsequent figures. Escape trajectory (continuous arc) is defined as the angle between wind direction (gray arrow) and the direction of motion (black arrow) of the escaping cockroach (black cockroach). Left and right stimuli were pooled as if each stimulus was always on the right side of the animal.

(C-G) The frequency distributions of five individual cockroaches tested in repeated trials. Numbers of responses are $93(C), 93$ (D), 89 (E), 81 (F), and 75 (G). The asterisk in (C) represents the trajectory of the escape response drawn in (B). Best-fit distributions in (C)-(G) are shown as multimodal curves. The statistics for the fitted curves are $(C)$ : Akaike weight $=0.91, \chi^{2}=14.77, p=0.254$, d.f. $=12 ;(D):$ Akaike weight $=0.93, \chi^{2}=19.87, p=0.099$, d.f. $=13 ;(E): A k a i k e$ weight $=0.85, \chi^{2}=8.98, p=0.623$, d.f. $=11 ;(F)$ : Akaike weight $=0.51, \chi^{2}=10.48, p=0.399$, d.f. $=10$; and $(G)$ : Akaike weight $=0.67, \chi^{2}=4.76, p=0.854$, d.f. $=9$. For all panels, concentric circles represent a frequency interval of 2 , bin intervals are $5^{\circ}$, and arrowheads indicate peaks, defined as those that contribute at least $5 \%$ to the best-fit curve.

Because the distributions of the ETs are not significantly different, all the data were pooled as a single data set (" $5 \mathrm{i}$," Figure 2A). The pooled data are significantly different from a uniform distribution both within $360^{\circ}$ [15] $\left(U^{2}\right.$ test; $p<$ $0.005 ; n=431$ ) and within an arc containing $99 \%$ of the data (see Supplemental Data) $\left(\chi^{2}=243.10 ; p<0.001\right.$; d.f. $=15$ ) and differ from a von Mises distribution (normal circular) $\left(U^{2}\right.$ test; $p<0.005 ; n=431$ ). The best-fit curve with the maximum likelihood method is a multipeak curve with peaks at $96.9^{\circ}$, $123.6^{\circ}, 150.3^{\circ}$, and $177.0^{\circ}$.

To determine whether the individual patterns observed above are representative of the variability in the population as a whole, we carried out a second series of experiments, in which 86 individuals were used, each startled only once ("singletons," Figure 2B). Again, the wind angles used were uniformly distributed (see Supplemental Data). The ET distribution of singletons (Figure 2B) and that of the $5 \mathrm{i}$ data set (Figure 2A) are not significantly different from each other $\left(\chi^{2}=5.25 ; p>0.25 ;\right.$ d.f. = 5; Figure 2$)$. The singleton data differ from a uniform distribution both within $360^{\circ}\left(U^{2}\right.$ test; $p<0.005$; $\mathrm{n}=86$ ) and within an arc containing $99 \%$ of the data (see Supplemental Data) $\left(\chi^{2}=29.49 ; p<0.005\right.$; d.f. = 11). Like the 5 i data set, the singleton data differ from a von Mises distribution $\left(U^{2}\right.$ test; $\left.p<0.025 ; n=86\right)$ and are also best fitted by a curve with peaks similar to those of the $5 \mathrm{i}$ data set, i.e., $93.3^{\circ}$, $120.8^{\circ}, 148.4^{\circ}$, and $175.9^{\circ}$ (Figure 2B). These results show that preferred ETs are not byproducts of different individual preferences within the population but are a shared characteristic of all animals from our inbred colony.
Analysis of the distributions of body-turn angles (within the range $0^{\circ}-120^{\circ}$, see Experimental Procedures) shows that they are uniformly distributed (singletons, $\chi^{2}=7.07 ; p>0.75$; d.f. $=11$; data set $5 i, \chi^{2}=13.98 ; p>0.1$; d.f. $=11$ ). Because the distribution of wind angles is also uniform, and ET is the sum of wind angle plus the body turn (see Figure 1A), the multimodal ETs cannot be due to any constraints (mechanical or neural) that restrict the cockroach to performing turns only at certain fixed angles relative to its body. This is confirmed by the distribution of the wind angles that elicit negligible turning $\left(0^{\circ}-10^{\circ}\right.$ body turn) (Figure $2 \mathrm{C}$ ). If cockroaches indeed prefer to escape along ETs at approximately $90^{\circ}, 120^{\circ}, 150^{\circ}$, and $180^{\circ}$ as we assert above, then these peaks should be found in the wind angles of escape responses in which cockroaches make little or no turning maneuver. Our results show that cockroaches escape approximately in line with their body direction only when stimulated at the predicted wind angles $\left(91.7^{\circ}\right.$, $119.7^{\circ}, 147.7^{\circ}$, and $175.7^{\circ}$; Figure $2 \mathrm{C}$ ), i.e., when they are already positioned along one of their preferred ETs (comparison between distributions in Figures $2 \mathrm{~A}$ and $2 \mathrm{C}, \chi^{2}=2.80, \mathrm{p}>0.5$, d.f. $=4$; comparison between distributions in Figures $2 \mathrm{~B}$ and 2C; $\chi^{2}=1.78, p>0.75$, d.f. $=4$ ).

To investigate any effect of wind angle on escape trajectory, we divided the $5 i$ data set into escape responses elicited by six different $30^{\circ}$ sectors of wind angle; the $0^{\circ}-30^{\circ}$ sector includes the most frontal stimulations, whereas the $150^{\circ}-180^{\circ}$ sector corresponds to the most posterior stimuli. The distributions of ETs divided into these six wind angle sectors are significantly different from one another $\left(\chi^{2}=104.45, p<0.001\right.$; 
A

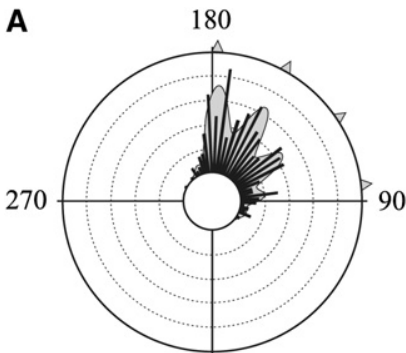

0,360

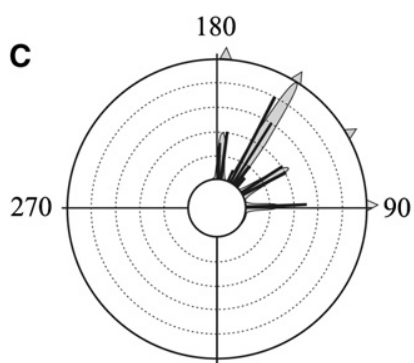

0,360
B

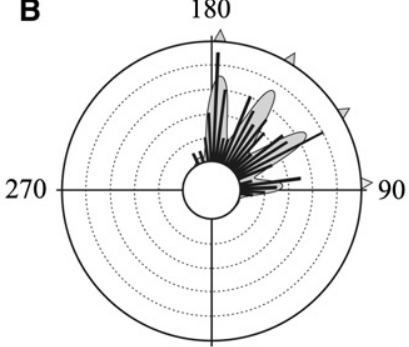

0,360

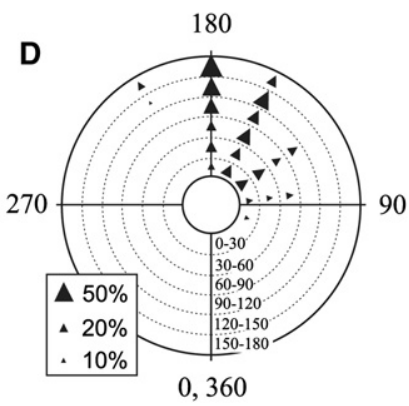

Figure 2. The ET Distributions of Five Individuals Tested in Repeated Trials and 86 Singletons Tested Each Once Only Are Not Significantly Different from One Another

(A) Frequency histograms of data set $5 i$ (pooling five individuals from Figure 1). Total number of responses $=431$, concentric circles represent a frequency of ten. Bin intervals are $5^{\circ}$. The best-fit curve Akaike weight $>0.99$ $\chi^{2}=23.71, p=0.536$, d.f. $=25$.

(B) Frequency histograms of singletons. Total number of responses $=86$, concentric circles represent a frequency of two. Bin intervals are $5^{\circ}$. The best-fit curve Akaike weight $=0.74, \chi^{2}=5.48, p=0.906$, d.f. $=11$.

(C) The distribution of wind angles in escape responses (data set $5 \mathrm{i}$ ) that showed little or no body turns $\left(0^{\circ}-10^{\circ}\right.$; number of responses $\left.=38\right)$. Concentric circles represent a frequency of two. Bin intervals are $5^{\circ}$. The wind angles show four peaks as in the preferred ETs found in $(A)$ and $(B)$; see text for details. The best-fit curve Akaike weight $=0.83, \chi^{2}=0.38, p=0.943$, d.f. $=3$. For all panels, best-fit distributions are shown as multimodal curves and arrowheads indicate peaks, defined as those that contribute at least $5 \%$ to the best fit curve.

(D) The effect of wind direction on ETs. Diagrammatic representation of the effect of wind angle on ET probability for the five individuals (data set 5i). Stimulus wind angle is indicated by the radial scale. The different ET peaks determined above for the $5 \mathrm{i}$ data are indicated by arrowheads at the appropriate angular positions, with the width of the arrowhead indicating the percentage contribution of that particular peak to the ET distribution at that particular wind angle. For scale, arrowheads are shown corresponding to $10 \%$, $20 \%$, and $50 \%$ contributions (inset). Peaks with a contribution of less than $5 \%$ are not shown. As wind angles increase, the lower value ET peaks (i.e., at approximately $90^{\circ}$ and $120^{\circ}$ ) disappear and a $204^{\circ}$ peak appears.

d.f. = 20; Figure 2D), showing that wind angle does have an effect on ET. The first four peaks present in Figure 2A reappear here for small wind angles, i.e., $<90^{\circ}$ (wind directed toward the head). However, as wind angles increase, the lower peaks disappear and an upper peak (at 204 ) appears so that, for wind directed at the rear of the animal, only three peaks are present (Figure 2D). In other words, because most escape responses are a turn away from the stimulus [6], the angles of ETs are almost always larger than those of wind angle. As a consequence, small ETs are used only when cockroaches are stimulated at small wind angles, whereas larger ETs (e.g., $177^{\circ}$ ) are used at all wind angles. Accordingly, the four main peaks found in the $5 i$ data set occur with different frequencies $\left(\chi^{2}=65, p<0.001\right.$; d.f. $=3$; Figure $\left.2 A\right)$.

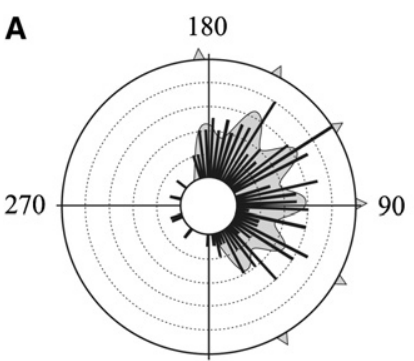

0,360

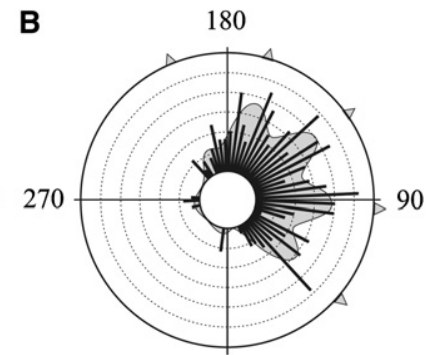

0,360
Figure 3. Re-analysis of Cockroach Escape-Behavior Data from the Published Literature Reveals Hitherto-Undetected Patterns of ET Distributions (A) Concentric circles represent a frequency of two. Bin intervals are $5^{\circ}$. Number of responses $=161$. Results are based on published data [6]. Akaike weight $=0.68, \chi^{2}=13.06, p=0.907$, d.f. $=21$.

(B) Concentric circles represent a frequency of 2.5 . Bin intervals are $5^{\circ}$. Number of responses $=253$. Results are based on published data [10] Akaike weight $=0.76, \chi^{2}=27.19, p=0.709$, d.f. $=32$. For both panels, the best-fit distribution is shown as a multimodal curve and arrowheads indicate peaks, defined as those that contribute at least $5 \%$ to the best-fit curve.

Our pooled data from the five individuals (data set $5 \mathrm{i}$, Figure 2A) were compared to data from previous studies [6, $10]$ of cockroach escape behavior with high sample sizes, originally analyzed with linear $x-y$ plots of body turn against wind angle, but reanalyzed here with circular statistics (see Supplemental Data). Our analyses show that the circular distributions of these previous data are nonuniform both within $360^{\circ}$ (Figure 3A, $U^{2}$ test; $p<0.005, n=161$; Figure $3 B, U^{2}$ test; $p<$ $0.005, n=253$ ) and within an arc containing $99 \%$ of the data (see Supplemental Data) (Figure 3A, $\chi^{2}=87.36$; $p<0.001$; d.f. = 28; Figure $3 B, \chi^{2}=159.92 ; p<0.001$; d.f. $=27$ ) and are different from a von Mises distribution (Figure $3 \mathrm{~A}, U^{2}$ test; $p<0.01, n=161$; Figure 3B, $U^{2}$ test; $p<0.01, n=253$ ). These data are also best fitted by curves with multiple, equally spaced peaks of equal width, at $31^{\circ}, 61^{\circ}, 92^{\circ}, 122^{\circ}, 152^{\circ}$, $183^{\circ}$, and $206^{\circ}$ (Figure $3 \mathrm{~A}$ ) and at $50^{\circ}, 88^{\circ}, 126^{\circ}, 164^{\circ}$, and $201^{\circ}$ (Figure 3B). Therefore, by reanalyzing these data as escape trajectories relative to the stimulus, we reveal the unpredictable, yet nonrandom, nature of this behavior. The multiple ET strategy provides an explanation for the wide range of body turns seen in $x-y$ plots, which has to date been explained by postulating a large amount of random "noise" in the system [16].

Comparison of the data obtained in the present study (Figure 2A) with the previous studies [6,10] (Figure 3) shows that the distributions of multiple peaks of ETs are significantly different (comparison with Figure $3 A, \chi^{2}=136$; d.f. $=8$; $p<$ 0.001 ; comparison with Figure $3 B, \chi^{2}=160.1$; d.f. $=9$; $p<$ 0.001 ). However, all three distributions do show multiple peaks of ETs; it is possible that different ET peaks occur in cockroaches from different laboratory colonies. Variation in the distributions could also be due to methodological differences of the behavioral experiment (size of arena, characteristics of the wind stimulus, walking versus standing still, etc.). Some of the difference between our data and previous work can be ascribed to differences in wind angle; whereas in our study wind angles are uniformly distributed, in previous work [6, 10] they are non uniform (see Supplemental Data) with a high proportion of stimulations toward the head. This would result in a higher proportion of ETs at smaller angles (see Figure 2D), as observed in previous work $[6,10]$ compared to our own. Notably, most ETs observed in Figures 2 and 3 are within the 
$90^{\circ}-180^{\circ}$ sector, which corresponds to the theoretically optimum range $[17,18]$.

The finding that cockroaches show multiple, discrete ETs has implications both in terms of the neural mechanisms that generate them and the resultant adaptive significance of multiple ETs. Adult cockroaches possess hundreds of wind-sensory hairs on their paired abdominal cerci that detect the direction of hair movements [6, 19-21]. The sensory neurons of these hairs input to giant interneurons (GIs) in the terminal ganglion, each with its own directional selectivity to wind [22]. A relatively small number of Gls convey this information to the thoracic ganglia, which produce the body turns [22]. The angular resolution of the sensory system must have a limit; however, this will simply add to the width of the peaks in the ET distribution. A poor resolution of wind direction would thus tend to smooth out, rather than sharpen, ET peaks.

Our results suggest that ETs are generated by a geocentric mechanism, i.e., in which the trajectories are fixed relative to an external reference (the stimulus), rather than by an egocentric mechanism in which trajectories are generated relative to a body reference (i.e., by fixed-body-turn angles). The neural mechanism for generating these multiple ETs is completely unknown, although it could involve the many thoracic interneurons that are interposed between the Gls and the motorneurons [23, 24], dorsal Gls, or even other wind-sensitive interneurons. Clearly, the finding of preferred ETs underscores the need to revisit the neural mechanisms controlling escape directions in the cockroach and other similar animal models. In addition, the experimental approach used here could be applied to animals from other taxa because high variability in escape directions is a common feature of many escape systems [3]. This would allow the construction of a more general theory of how animal generate unpredictable escape trajectories.

Independently of how the multiple ETs are generated, what matters in terms of adaptive significance is that the resultant escape behavior is sufficiently unpredictable (i.e., protean [3]) to predators that a selective advantage is achieved. It is unlikely that there is any particular fitness significance for any specific ET, i.e., escaping at $120^{\circ}$ or $150^{\circ}$, but not at $135^{\circ}$. Although each peak must emerge from the neuronal computation of the escape-behavior circuit, the evolutionary significance of the multiple ETs is likely to lie in the overall multimodal pattern, which allows an animal to generate the variability necessary to keep the predator "guessing."

\section{Experimental Procedures}

\section{Experimental Measurements}

Animal keeping and the experimental apparatus are described in the Supplemental Data. Wind angles were measured with respect to the animal's midline in the frame preceding the onset of the wind stimulus. All individuals were walking at the time of stimulation. Escapes from left and right stimuli were pooled as if the stimuli were always from the right; no asymmetries were present in the $5 \mathrm{i}$ data set when this was divided into escapes that were stimulated from the left and right sides of the animals $\left(\chi^{2}=6.01 ; p>0.25\right.$; d.f. $=6$; data not shown). Thus wind angle spanned $0^{\circ}-180^{\circ}$, where head-on stimulations were designated as $0^{\circ}$ and stimulations directly from the rear corresponded to $180^{\circ}$. Escape trajectories (ETs) were defined as the angle between the direction of motion at the end of the turning component of the escape and the direction of the stimulus (Figure 1A). ETs therefore correspond to the sum of the wind angle and the body turn by the cockroach (defined as the angle between the body's midline before stimulation and its direction of motion at the end of the response), in which body turns away from the stimulus bear a positive sign and body turns toward the stimulus bear a negative sign. Therefore, ETs in a direction directly away from the stimulus were at $180^{\circ}$. ETs $>180^{\circ}$ indicated that animals had turned through a greater angle than was required to face directly away from the stimulus (see Figure 1). ETs potentially spanned $360^{\circ}$ and were treated as a circular variable [15].

\section{Statistics}

Goodness-of-fit tests against uniform and von Mises (normal circular [15]) distributions for ET data were carried out with $U^{2}$ tests [15] with Oriana circular statistics software. We used $\chi^{2}$ tests to compare distributions [25] with data divided into bins. Wind-angle data were divided into nine $20^{\circ}$ bins $\left(0^{\circ}-20^{\circ} ; 20^{\circ}-40^{\circ}\right.$, and so on). ETs data were divided into eighteen $20^{\circ}$ bins, centered at $0^{\circ}, 20^{\circ}, 40^{\circ}$, etc. Any bin with expected frequencies $<5$ was pooled with the adjacent bin until an expected frequency $>5$ was reached [25]. Uniformity of distribution in the body-turn angles was analyzed in singletons and data set $5 \mathrm{i}$ with $\chi^{2}$ tests. Body-turn data were divided into $10^{\circ}$ bins. To avoid low frequencies at the tail end of the distribution, we analyzed body-turn data within the range $0^{\circ}-120^{\circ}$, which comprised $86.8 \%$ of all turns for data set $5 i$ and $96.25 \%$ for the singletons. Wind-angle distribution and additional statistics are described in the Supplemental Data.

\section{Supplemental Data}

Supplemental Data include Supplemental Experimental Procedures and can be found with this article online at http://www.current-biology.com/ supplemental/S0960-9822(08)01340-7.

\section{Acknowledgments}

P.D. was supported by the CNR - Consiglio Nazionale delle Ricerche, D.B. was supported by a BBSRC Studentship, J.M.B. was supported by NIHSCORE S06 GM0088224 and RCMI G12 RR03051, J.P.B. was supported by a BBSRC grant. We thank U. Muller, R. Blanco, C. Comer, D. Osorio, and T. Collett and five anonymous referees for insightful comments.

Received: July 16, 2008

Revised: September 12, 2008

Accepted: September 26, 2008

Published online: November 13, 2008

\section{References}

1. Bullock, T.H. (1984). Comparative neuroethology of startle, rapid escape and giant fiber-mediated responses. In Neural Mechanisms of Startle Behaviour, R.C. Eaton, ed. (New York: Plenum Press), pp. 1-13.

2. Ydenberg, R.C., and Dill, L.M. (1986). The economics of fleeing from predators. Adv. Stud. Behav. 16, 229-249.

3. Humphries, D.A., and Driver, P.M. (1970). Protean defence by prey animals. Oecologia 5, 285-302.

4. Driver, P.M., and Humphries, D.A. (1988). Protean Behaviour: The Biology of Unpredictability (Oxford: Clarendon).

5. Godin, J.-G.J. (1997). Evading predators. In Behavioural Ecology of Teleost Fishes, J.-G.J. Godin, ed. (Oxford: Oxford University Press), pp. 191-236.

6. Camhi, J.M., and Tom, W. (1978). The escape behaviour of the cockroach Periplaneta americana. 1. Turning response to wind puffs. J. Comp. Physiol. [A] 128, 193-201.

7. Eaton, R.C., Lavender, W.A., and Wieland, C.M. (1981). Identification of Mauthner initiated response patterns in goldfish: Evidence from simultaneous cinematography and electrophysiology. J. Comp. Physiol. [A] 144, 521-531.

8. Domenici, P., and Blake, R.W. (1993). Escape trajectories in angelfish (Pterophyllum eimekei). J. Exp. Biol. 177, 253-272.

9. Camhi, J.M. (1983). Neuroethology: Nerve Cells and the Natural Behavior of Animals (Sunderland: Sinauer).

10. Comer, C.M., and Dowd, J.P. (1987). Escape turning behaviour of the cockroach. Changes in directionality induced by unilateral lesions of the abdominal nervous system. J. Comp. Physiol. 160, 571-583.

11. Ritzmann, R.E., and Pollack, A.J. (1988). Wind-activated thoracic interneurons of the cockroach: II. Patterns of connection from ventral giant interneurons. J. Neurobiol. 19, 589-611.

12. Stierle, I.E., Getman, M., and Comer, C.M. (1994). Multisensory control of escape in the cockroach Periplaneta Americana. 1. Initial evidence from patterns of wind-evoked behaviour. J. Comp. Physiol. 174, 1-11. 
13. Stern, M., Ediger, V.L., Gibbon, C.R., Blagburn, J.M., and Bacon, J.-P. (1997). Regeneration of cercal filiform hair sensory neurons in the firstinstar cockroach restores escape behavior. J. Neurobiol. 33, 439-458.

14. Rinberg, D., and Davidowitz, H. (2000). Do cockroaches 'know' about fluid dynamics? Nature 405, 756.

15. Fisher, N.I. (1993). Statistical Analysis of Circular Data (Cambridge: Cambridge University Press).

16. Ezrachi, E.A. (2003). Computational model of the cockroach escape behavior: Winner and losers in a population code. Biol. Cybern. 88, 33-45.

17. Weihs, D., and Webb, P.W. (1984). Optimal avoidance and evasion tactics in predator-prey interactions. J. Theor. Biol. 106, 189-206.

18. Domenici, P. (2002). The visually-mediated escape response in fish: Predicting prey responsiveness and the locomotor behaviour of predators and prey. Mar. Freshwat. Behav. Physiol. 35, 87-110.

19. Westin, J. (1979). Responses to wind recorded from the cercal nerve of the cockroach Periplaneta americana. I. Response properties of single sensory neurons. J. Comp. Physiol. [A] 133, 97-102.

20. Thompson, K.S.J., Blagburn, J.M., Gibbon, C.R., and Bacon, J.P. (1992). Correlation of filiform hair position with sensory afferent morphology and synaptic connections in the second instar cockroach. J. Comp. Neurol. 320, 213-227.

21. Kondoh, Y., Arima, T., Okuma, J., and Hasegawa, Y. (1991). Filter characteristics of cercal afferents in the cockroach. J. Comp. Physiol. [A] 169, 653-662.

22. Kolton, L., and Camhi, J.M. (1995). Cartesian representation of stimulus direction: Parallel processing by two sets of giant interneurons in the cockroach. J. Comp. Physiol. [A] 176, 691-702.

23. Westin, J., Ritzmann, R.E., and Goddard, D.J. (1988). Wind-activated thoracic interneurons of the cockroach: I. Responses to controlled wind stimulation. J. Neurobiol. 19, 573-588.

24. Ritzmann, R.E., and Pollack, A.J. (1990). Parallel motor pathways from thoracic interneurons of the ventral giant interneuron system of the cockroach, Periplaneta americana. J. Neurobiol. 21, 1219-1235.

25. Batschelet, E. (1981). Circular Statistics in Biology (New York: Academic Press). 